\title{
STEGANOGRAPHY TECHNIQUES: A SURVEY
}

Sachin(Correspondence Author $)^{1} \&$ Sunita Dhingra ${ }^{2}$

Abstract-With the growth in network technology, there is parallel growth of information being exchange over the network environment which in turn also demands the security of information to maintain its confidentiality along with integrity of data. Many of technologies are present such as cryptography and steganography to achieve the above purpose. In this paper a number of image steganography techniques are presented along with their review and the widely used metrics for image quality such as PSNR and MSE are discussed also.

Keywords: Steganography, Cryptography, GLM, LSB, PIT, PSNR and MSE.

\section{INTRODUCTION}

Escalation in communication in modern world of digital communication also escalates the demand of security of data to be communicated. Application of security like protection of copyrights using watermarking or finger printing for any of digital media like videos, audios and images $[1,2,3]$. Two major techniques that dominate the domain of security are cryptography and steganography. While considering the former technique, it may give a chance to suspect on secured data but on the other hand the latter one may not even give a single chance of secured data depending upon perceptibility [4]. Perceptibility refers to the level of vision. If a good design procedure is used then it is imperceptible/indistinguishable otherwise secured data can be seen either closely observing it or it is blatantly obvious [5,6]. A paradigm for steganography encompasses a cover medium $(\mathrm{H})$, information to be hidden (I) and a key (Y) that may indicate on which pixel information to be concealed as shown below:

Figure (1) - Embedding (Em) Paradigm

Where $\mathrm{F}(\mathrm{H}, \mathrm{I}, \mathrm{Y})$ may use different steganographic techniques for concealing information in cover medium.

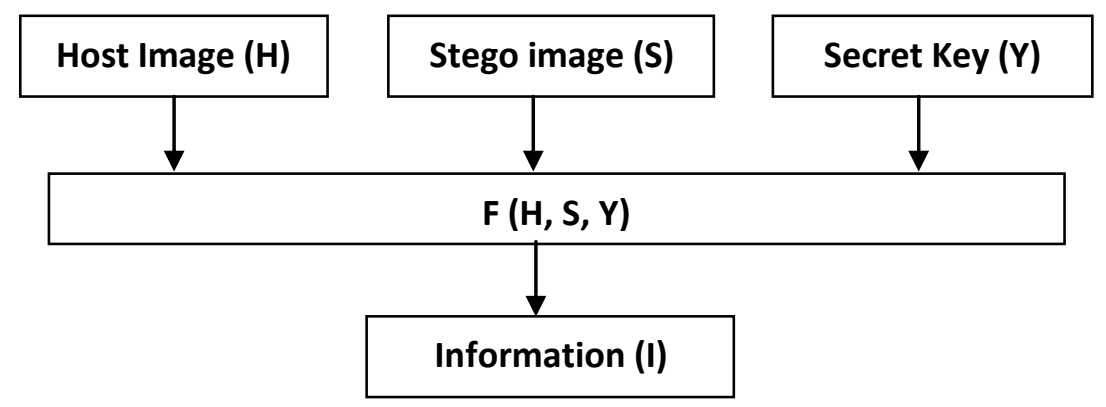

Figure (2) - Extraction (Ex) Paradigm

Mathematically it can be reported as:

$\mathbf{E m}: \mathbf{H} \diamond \mathbf{I} \diamond \mathbf{Y} \rightarrow \mathbf{S}$

Ex: $\mathbf{H} \diamond \mathbf{S} \diamond \mathbf{Y} \rightarrow \mathbf{I}$

$\checkmark:$ Procedure used for Embedding (Em) and Extraction (Ex) [7].

While choosing an embedding procedure it is always focused on: Security, Payload Capacity and Robustness. Security refers to level of extraction of hidden data from an unauthorized access. Higher the level of extraction, higher will be security [8,9]. Payload refers to the capacity of data that can be concealed in a cover object and the third factor is robustness which refers amount of data obtained after manipulation of stego object i.e resilience of concealed information [10]. There is always tradeoff between security (Imperceptibility), payload and robustness as shown

\footnotetext{
${ }^{1}$ Department of Computer Science and Engineering, University Institute of Engineering and Technology Maharshi Dayanand University, Rohtak-124001 Haryana, INDIA

${ }^{2}$ Department of Computer Science and Engineering, University Institute of Engineering and Technology Maharshi Dayanand University, Rohtak-124001 Haryana, INDIA
} 


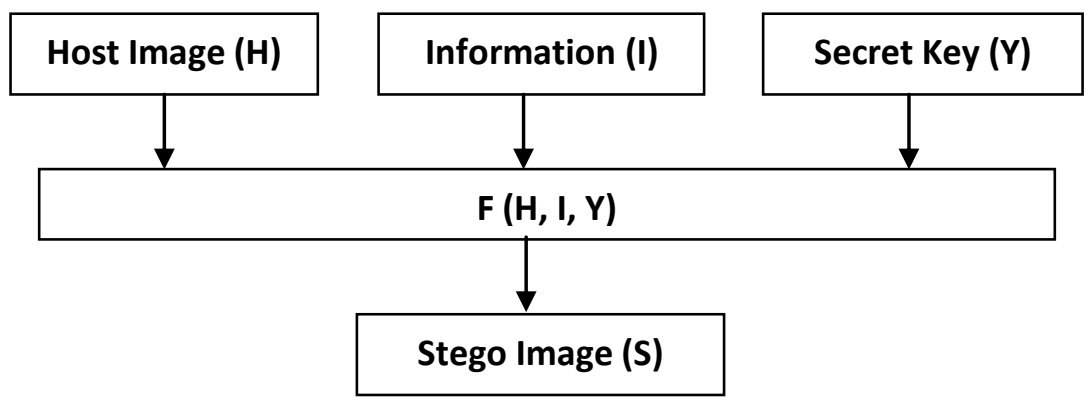

Fig (3) Trade-off Triangle [11, 12]

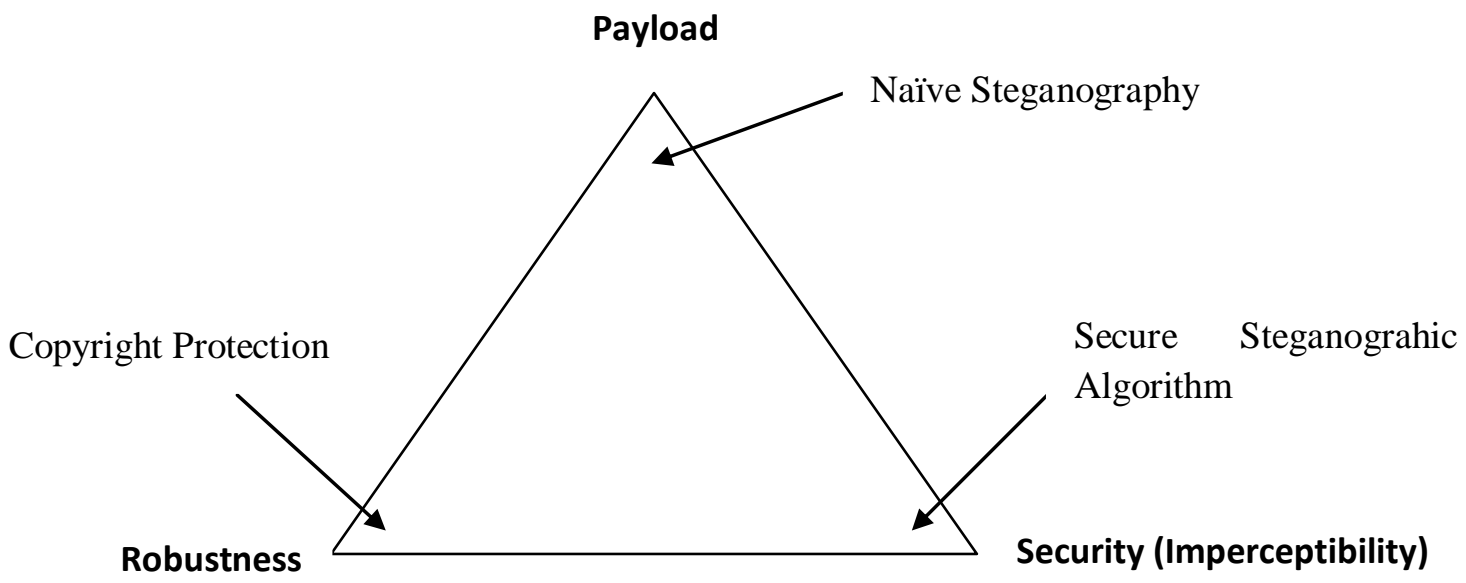

\section{POPULAR TECHNIQUES FOR IMAGE STEGANOGRPAHY}

Today a number of image steganography techniques are present for secure communication; some of them are used with some modification in their original behaviour either by enhancing it or by combining it with some other secure algorithm while others can be used without any modification. Some of popular steganography algorithms are discussed below:

\subsection{LSB Approach [13,14]}

It is most popularly known technique for its simplicity and ability to provide better results with high degree of imperceptibility. Actually, this approach works by embedding the secret message bits at the LSB of every pixel of cover medium.

\section{Advantages:}

- Implementation is very easy.

- Invisibility to human visual system.

\section{Disadvantages:}

- Easily vulnerable to attacks

- Resilience to noise.

- Not effected by compression techniques.

\subsection{Gray Level Modification (GLM) Techniques [ 15,16]}

GLM is a mapping technique wherein gray values of pixels of an image is modified either by considering the odd or even pixels. Depending upon choice if all odd pixels are chosen; then these are converted into even gray levels by decrementing its value by one and message bits are embedded over them.

Advantages:

- Optimal chances of embedding.

\section{Disadvantages:}

- Implementation is very simple.

- Vulnerable to attacks.

- Effected by noise 
- Also vulnerable to compression.

\subsection{Pixel Indicator Technique (Pit) [ 17, 18 ]}

PIT is an image steganography model wherein a colored (24 - bits) host image is taken in which every pixel is divided in three channels i.e R, G and B. R channel used as indicator channel to indicate location of data on $\mathrm{G}$ and $\mathrm{B}$ channels.

\section{Advantages:}

\section{Disadvantages:}

- More payload capacity.

- Higher security than LSB

- Security can be compromised with little efforts.

\subsection{Transform Domain Techniques [19, 20 ]}

This technique is more resilient to image manipulation such as cropping, image contrasting, compression etc. In this, cover image is partitioned in different bands to hide data. Such techniques are DCT, DFT and DWT etc.

\section{POPULAR METRICS OF IMAGE QUALITY}

PSNR refers to Peak Signal To Noise Ratio used to find the quality of an image for matching the similarity between stego and cover image. It is evaluated in terms of decibels $(\mathrm{dB})$ and its mathematical equation is given as:

Where I: Peak value of Image intensity which is 255 [51].

$$
P S N R=10 \log _{10}\left[\frac{I^{2}}{M S E}\right]
$$

MSE refers to Mean Square Error. It is inversely proportional to PSNR and it is given as

$$
M S E=\frac{1}{[I * J]} \sum_{m=1}^{J} \sum_{n=1}^{I}\left(P_{m n}-Q_{m n}\right)^{2}
$$

Where I and $\mathbf{J}$ represent rows and columns of an Image respectively and $\mathrm{P}_{\mathrm{mn}}, \mathrm{Q}_{\mathrm{mn}}$ are intensity of $\mathrm{mn}^{\text {th }}$ pixel of cover and stego image respectively [52].

\section{LITERATURE SURVEY}

This paper provides a detailed review of different approaches used in steganoraphy in recent years. Table 1 provides the extensive survey of researches; with the name of author, year of publication in descending order of research along with purposed work and approaches used by them as shown below:

Table - I

\begin{tabular}{|l|l|l|}
\hline Authors & Year & Proposed Work \\
\hline Devi et. al [21] & 2017 & $\begin{array}{l}\text { Proposed a novel approach to prevent from phishing attack by using RGB images. That } \\
\text { further undergoes for compression using Discrete Consine Transform technique. }\end{array}$ \\
\hline Avci et. al [22] & 2016 & $\begin{array}{l}\text { Presented a wavelet transform based steganographic approach which shows good } \\
\text { imperceptibility and less distortion of cover-object. }\end{array}$ \\
\hline Jain \& lenka [23] & 2016 & $\begin{array}{l}\text { Presented steganographic approach that used Rabin Cryptosystem for encryption of } \\
\text { secret data and outputs it into various partitions which are dynamically embedded into } \\
\text { diagonal queue. }\end{array}$ \\
\hline Singh et. al.[24] & 2016 & $\begin{array}{l}\text { Proposed multiple watermarking based approach in which cover-object can conceal } \\
\text { multiple watermarks using frequency domain steganographic technique in which sub } \\
\text { bands of cover image are deinterlaced on which watermarks are embedded. }\end{array}$ \\
\hline Joshi et. al [25] & 2016 & $\begin{array}{l}\text { Analysed a spatial domain technique; LSB in terms of PSNR and MSE based metrics } \\
\text { for image quality by considering images of different dimension over the data of different } \\
\text { sizes. }\end{array}$ \\
\hline Joshi et.al [26] & 2016 & $\begin{array}{l}\text { Proposed a model using Gray Level modification based steganographic approach in } \\
\text { medical system. In this a pixel is made suitable by adding one or subtracting one from } \\
\text { pixel value so that information bit can be adjusted on it to get high degree of } \\
\text { imperceptibility. }\end{array}$ \\
\hline Joshi et. al [27] & 2016 & $\begin{array}{l}\text { Used LSB approach in image steganography for hiding watermark image and further an } \\
\text { analysis is made on adding salt \& pepper noise in this stego image to check the }\end{array}$ \\
\hline
\end{tabular}




\begin{tabular}{|c|c|c|}
\hline & & robustness of resultant image. \\
\hline Allawadhi [28] & 2016 & $\begin{array}{l}\text { A cyclic LSB approach used in a RGB- image wherein insertion of message bit taken } \\
\text { place in cyclic manner. Initially, } 1^{\text {st }} \text { pixel's R component taken as host, then } 2^{\text {nd }} \text { pixel's G } \\
\text { component and so on until message is embedded in host image. Further the result are } \\
\text { analysed using PSNR and MSE based parameters. }\end{array}$ \\
\hline Allawadhi [29] & 2016 & $\begin{array}{l}\text { Proposed PIT image steganography model wherein host image is taken as coloured } \\
\text { image so every pixel is divided in three channels i.e R, G and B wherein R channel used } \\
\text { as indicator channel to indicate location of data on } G \text { and B channels and at last results } \\
\text { are analysed in terms of PSNR and NCC based factors. }\end{array}$ \\
\hline Tang et. al [30] & 2015 & $\begin{array}{l}\text { Proposed a steganography model for voice over internet protocol (VOIP). Proposed } \\
\text { approach shows good results in terms of low PESQ rate and high payload capacity. }\end{array}$ \\
\hline Fa et. al [31] & 2015 & $\begin{array}{l}\text { Proposed a DCT steganalysis approach that used an additive operator which in turn } \\
\text { selects non-zero value for AC coefficients. These coefficients used as a carrier for secret } \\
\text { message to be embedded with. }\end{array}$ \\
\hline Choi \& Pun [32] & 2015 & $\begin{array}{l}\text { Provided a digital watermarking method in spatial domain using integer transform based } \\
\text { approach. PSNR, Payload capacity and Robustness of given approach is analysed and it } \\
\text { is found that for a watermark of } 100 \text { bits in } 512 * 512 \text { greyscale image, the obtained } \\
\text { PSNR was over } 60 \mathrm{~dB} \text { which is quite satisfactory. }\end{array}$ \\
\hline Husein \& Badi [33] & 2014 & $\begin{array}{l}\text { Used two phased simulation package as Artificial neural network where } 1^{\text {st }} \text { phase asks } \\
\text { for embedding and } 2^{\text {nd }} \text { phase to read the concealed message and used Levenberg- } \\
\text { Marquardt (LM) also for training the N.N }\end{array}$ \\
\hline $\begin{array}{l}\text { Afrakhteh et. al. } \\
{[34]}\end{array}$ & 2014 & $\begin{array}{l}\text { Proposed a spatial domain steganographic technique that uses image quality factor for } \\
\text { finding pixels used for embedding the secret data. This technique increases payload } \\
\text { capacity up to } 4 \text { bits per pixel (bpp) and still shows good PSNR (Peak Signal to Noise } \\
\text { Ratio) value. }\end{array}$ \\
\hline $\begin{array}{l}\text { Tayel \& Shawky } \\
\text { [35] }\end{array}$ & 2014 & $\begin{array}{l}\text { Proposed a modified steganography approach using fuzzification over message, also } \\
\text { embedding strength factor (ESF) conceal in cover-image. Various evaluation metrics } \\
\text { like PSNR, Entropy and MSE are used for analysis purpose. }\end{array}$ \\
\hline Liu et.al. [36] & 2014 & $\begin{array}{l}\text { Non-overlapping blocks of } 4 * 4 \text { sizes are obtained after partitioning cover image. } \\
\text { Likewise Watermark is also divided into } 4 \text { equal blocks, before this division watermark } \\
\text { image is encrypted using Arnold Transform. Further watermark blocks are embedded } \\
\text { into host-image's blocks; which proves to be robust approach for watermarking. }\end{array}$ \\
\hline Jung \& Yoo [37] & 2014 & $\begin{array}{l}\text { Used a steganography approach that uses interpolation technique. Further, LSB based } \\
\text { approach used to hide the information in the resultant cover-image. }\end{array}$ \\
\hline Choi et. al [38] & 2012 & $\begin{array}{l}\text { Presented a data fusion procedure using steganography techniques wherein they used } \\
\text { advertisement image for information gathering by converting it into a gateway. }\end{array}$ \\
\hline $\begin{array}{ll}\text { C. } & \text { Oluwakemial } \\
\text { [39] } & \end{array}$ & 2012 & $\begin{array}{l}\text { Proposed a combined cryptography and steganography based information hiding } \\
\text { technique in which an audio file used as an cover-medium, which used LSB based } \\
\text { approach to embed message in it. }\end{array}$ \\
\hline $\begin{array}{l}\text { Farschi \& Farschi } \\
{[40]}\end{array}$ & 2012 & $\begin{array}{l}\text { Used chaotic approach for hiding information. Both sender and receiver settle on a secret } \\
\text { key for secure communication. }\end{array}$ \\
\hline Jung \& Yoo [41] & 2012 & $\begin{array}{l}\text { This paper provides a combination of interpolation and edge detection based information } \\
\text { hiding algorithm. Interpolation method scales up cover-image size to increase payload } \\
\text { length whereas edge detection method enhances the quality of resultant (Stego) image } \\
\text { Experiments shows good PSNR \& quality Index. }\end{array}$ \\
\hline $\begin{array}{l}\text { Ataby \& Naima } \\
{[42]}\end{array}$ & 2010 & $\begin{array}{l}\text { Presented a wavelet transform based steganographic approach which shows good } \\
\text { imperceptibility and less distortion of cover-object. }\end{array}$ \\
\hline Gutub & 2010 & ixel indicator steganographic technique works on 24 bit pixel images i.e RGB \\
\hline
\end{tabular}




\begin{tabular}{|l|l|l|}
\hline [43] & & $\begin{array}{l}\text { images wherein every pixel has 3- Channels (R,G \& B) out of which 1-channel is used } \\
\text { as Indicator \& other 2 for embedding secret message. The proposed method not only } \\
\text { increases the payload capacity but also security. }\end{array}$ \\
\hline Luo et. al [44] & 2010 & $\begin{array}{l}\text { Presented a modified scheme of pixel value differencing. In this, cover-object is divided } \\
\text { into squared blocks and each of them can be rotated at random degree. Further obtained } \\
\text { image is divided in such a way that middle pixel is used for embedding secret out of } \\
\text { three continuous pixel. }\end{array}$ \\
\hline Cheddad et. al [45] & 2009 & $\begin{array}{l}\text { Proposed review of different steganographic techniques along with their analysis and } \\
\text { further recommended to use object oriented technology for embedding }\end{array}$ \\
\hline Yu et. al [46] & 2008 & $\begin{array}{l}\text { Used an enhanced version of original LSB approach named Plus Minus 1 (PM1) over } \\
\text { JPEG images using genetic algorithms based approach. Proposed method increase } \\
\text { capacity as well as security of data. }\end{array}$ \\
\hline $\begin{array}{l}\text { Bailey \& Curran } \\
\text { [47] }\end{array}$ & 2006 & $\begin{array}{l}\text { Analysed 7 - different steganography techniques after implementing them. All of them } \\
\text { used least significant bit (LSB) manipulation based approach and analysed in terms of } \\
\text { capacity of payload \& resilience to different types of attacks. }\end{array}$ \\
\hline Chen \& Lin [48] & 2006 & $\begin{array}{l}\text { Used frequency domain (Discrete Wavelet Transform) based steganographic approach. } \\
\text { In which some operation are applied on secret data before concealing. }\end{array}$ \\
\hline Wu \& Tsai [49] & 2003 & $\begin{array}{l}\text { Used PVD technique for information hiding in which cover image is divided into blocks } \\
\text { that are non-overlapping having 2 pixels that are consecutive and each block's pixel } \\
\text { difference value is used to embed secret data. This scheme presents better results than } \\
\text { classical LSB approach. }\end{array}$ \\
\hline
\end{tabular}

\section{CONCLUSION}

A survey of different steganography approaches is presented of recent years. It is found that most of the techniques used LSB; spatial domain techniques while some of the others used frequency domain techniques such as DWT and DCT. A more robust approach can be used by combining these two techniques wherein an image can be subdivided in four bands using frequency domain technique and now the data can be concealed in any of four sub bands using a spatial domain technique further the required sub band can be selected based on some key that adds more security to this approach.

\section{REFERENCES}

[1] N. F. Johnson and S. Jajodia, "Exploring steganography: Seeing the unseen," Computer (Long. Beach. Calif)., vol. 31, no. 2, pp. 26-34, 1998.

[2] M. Kaur, "Cryptography as a Pedagogical Tool," Primus, vol. 18, no. 2, pp. 198-206, 2008.

[3] R. J. Anderson, F-. A. P. Petitcolas, On the limits of steganography, IEEE Journal of Selected Areas in Communications, Special Issue on Copyright and Privacy Protection, 16(1998)4, 474-481.

[4] N. F. Johnson, S. Jajodia, Steganalysis of images created using current steganography software, Lecture Notes in Computer Science, Vo1.1525, Springer-Verlag, Berlin, 1998, 48-61.

[5] Lee Y-P, Lee J-C, ChenW-K, ChangK-C, Su I-J, Chang C-P (2012)High-payload image hidingwith quality recovery using tri-way pixel-value differencing. Inf Sci 191:214-225

[6] Mielikainen J (2006) LSB matching revisited. Signal Proc Lett IEEE 13:285-287

[7] Westfeld A, Pfitzmann A (2000) Attacks on steganographic systems. In: Information Hiding. pp 61-76

[8] J. Fridrich, "Feature-Based Steganalysis for JPEG Images and its Implications for Future Design of Steganographic Schemes", in Proc. 6th Int. Workshop on Information Hiding, Toronto, Canada, 23-25 May 2004, pp. 67-81

[9] J. Fridrich, M. Goljan, and D. Hogea, "Steganalysis of JPEG Images: Breaking the F5 Al- gorithm", in Proc. 5th International Workshop on Information Hiding, Noordwijkerhout, The Netherlands, 79 Oct. 2002, pp. 310 - 323.

[10] R.A. Johnson, "Miller\&Freund's Probability and Statistics for Engineers", Prentice Hall of India Pvt. Ltd., New Delhi, 2003

[11] K. Solanki, N. Jacobsen, U. Madhow, B.S. Manjunath, and S. Chandrasekaran, "Robust image-adaptive data hiding based on erasure and error correction", IEEE Trans. on Image Processing, vol. 13, no. 12, Dec. 2004, pp. 1627-1639.

[12] Wu, M., Liu, B., 2004. Data hiding in binary image for authentication and annotation. IEEE Transactions on Multimedia 6 (August), $528-538$ 
[13] Tseng, Y.C., Chen, Y.Y., Pan, H.K., 2002. A secure data hiding scheme for binary images. IEEE Transactions on Communications 50 (August), $1227-$ 1231.

[14] Provos, N. \& Honeyman, P., "Hide and Seek: An introduction to steganography", IEEE Security and Privacy Journal, 2003

[15] Bender, W., Gruhl, D., Morimoto, N. \& Lu, A., "Techniques for data hiding”, IBM Systems Journal, Vol 35, 1996

[16] Petitcolas, F.A.P., Anderson, R.J. \& Kuhn, M.G., "Information Hiding - A survey”, Proceedings of the IEEE, 87:07, July 1999

[17] Anderson, R.J. \& Petitcolas, F.A.P., "On the limits of steganography", IEEE Journal of selected Areas in Communications, May 1998

[18] A. A. A. Gutub, "Pixel indicator technique for RGB image steganography," J. Emerg. Technol. Web Intell., vol. 2, no. 1, pp. 56-64, 2010.

[19] Mamta Juneja and Parvinder Singh Sandhu, 'Designing of Robust Image Steganography Technique Based on LSB Insertion and Encryption', ARTCom 2009 - International Conference on Advances in Recent Technologies in Communication and Computing, 2009, 302-5

[20] K. Muhammad, M. Sajjad, I. Mehmood, S. Rho, and S. W. Baik, "A novel magic LSB substitution method (M-LSB-SM) using multi-level encryption and achromatic component of an image," Multimed. Tools Appl., vol. 75, no. 22, pp. 14867-14893, 2016.

[21] K. Suganya Devi, P.Srinivasan,M. Vaishnave, G. Arutperumjothi, "Secure E Pay System Using Stegnography and Visual Cryptography,” vol. 6, no. 7, pp. 269-272, 2017.

[22] E. Avci, T. Tuncer, and D. Avci, "A Novel Reversible Data Hiding Algorithm Based on Probabilistic XOR Secret Sharing in Wavelet Transform Domain,” Arab. J. Sci. Eng., vol. 41, no. 8, pp. 3153-3161, 2016

[23] Jain, M., Lenka, S. K. (2016). "Diagonal queue medical image steganography with Rabin cryptosystem. Brain Informatics", 3(1), 39-51, 2016

[24] S. Singh, R.K. Arya, H.Sharma, “A Robust Deinterlacing Multiple Image Watermarking Technnique in DWT”, 978-1-5090-0082-1, 2016

[25] K. Joshi, R. Yadav, S.Allwadhi, "PSNR and MSE Based Analysis of LSB Image Steganography in Spatial Domain", 978-1-5090-0082-1, 2016

[26] K. Joshi, S. Allwadhi, and R. Yadav, "Image Steganography Model for Medical Images Using GLM," Compusoft, vol. 5, no. 5, pp. $2120-2124,2016$.

[27] K. Joshi, R. Yadav, and S. Allwadhi, "Exploration of Least Significant Bit Based Watermarking and Its Robustness against Salt and Pepper Noise," vol. 10, no. 7, pp. 1400-1408, 2016.

[28] S. Allawadhi, “Analysis of Cyclic LSB Method Using PSNR and MSE," vol. 4, no. 7, pp. 18-24, 2016

[29] S. Allawadhi, "PSNR and NCC Based Evaluation of PIT," vol. 5, no. 1, pp. 6-13, 2016.

[30] S.Tang, Q.Chen, W. Ahang, Y.Huang, "Universal steganography model for low bit-rate speech codec", doi: 10, 1002/sec.1183, 2015.

[31] M. Jia-Fa, N. Xin-Xin, X. Gang, S. Wei-Guo, and Z. Na-Na, “A steganalysis method in the DCT domain,” Multimed. Tools Appl., vol. 75, no. 10, pp. 5999-6019, 2016.

[32] K. C. Choi and C. M. Pun, "Robust lossless digital watermarking using integer transform with Bit plane manipulation," Multimed. Tools Appl., vol. 75, no. 11, pp. 6621-6645, 2016.

[33] S. Husien and H. Badi, “Artificial neural network for steganography,” Neural Comput. Appl., vol. 26, no. 1, pp. 111-116, 2014.

[34] M. Afrakhteh, I. Moon, and J. A. Lee, "Double phase modular steganography with the help of error images," Multimed. Tools Appl., vol. 74, no. 13, pp. 4833-4847, 2015

[35] M. Tayel, “An Optimized Trade-off Decomposition Steganography Algorithm,” vol. 86, no. 5, pp. 19-23, 2014.

[36] J. Liu, G. Tang, and Y. Sun, "A secure steganography for privacy protection in healthcare system," J. Med. Syst., vol. 37, no. 2, 2013.

[37] K.-H. Jung and K.-Y. Yoo, "High-capacity index based data hiding method," Multimed. Tools Appl., vol. 74, no. April 2014, pp. 2179-2193, 2014.

[38] Y. H. Choi, D. Kim, S. Rho, and E. Hwang, "Converting image to a gateway to an information portal for digital signage," Multimed. Tools Appl., vol. 71, no. 1, pp. 263-278, 2014.

[39] A. Oluwakemi. C, A. Kayode S, O. Ayotunde J, "Efficient Data hiding System using Cryptography and Steganography", Vol - 4(11), 2012

[40] S. M. R. Farschi and H. Farschi, "A novel chaotic approach for information hiding in image," Nonlinear Dyn., vol. 69, no. 4, pp. 1525-1539, 2012.

[41] H. Jung and K.-Y. Yoo, "Three-Directional Data Hiding Method for Digital Images," Cryptologia, vol. 38, no. 2, pp. 178-191, 2012.

[42] A. Al-Ataby and F. Al-Naima, "A modified high capacity image steganography technique based on wavelet transform," Changes, vol. 7, no. 4, pp. 358364,2008

[43] A. A. A. Gutub, "Pixel indicator technique for RGB image steganography," J. Emerg. Technol. Web Intell., vol. 2, no. 1, pp. 56-64, 2010.

[44] X. Luo, F. Liu, C. Yang, S. Lian, and Y. Zeng, "Steganalysis of adaptive image steganography in multiple gray code bit-planes," Multimed. Tools Appl., vol. 57, no. 3, pp. 651-667, 2012

[45] A. Cheddad, J. Condell, K. Curran, and P. Mc Kevitt, "Digital image steganography: Survey and analysis of current methods," Signal Processing, vol. 90, no. 3, pp. 727-752, 2010.

[46] L. Yu, Y. Zhao, R. Ni, and Z. Zhu, "PM1 steganography in JPEG images using genetic algorithm," pp. 393-400, 2009.

[47] K. Bailey and K. Curran, An evaluation of image based steganography methods, vol. 30, no. 1. 2006.

[48] P. Chen and H. Lin, “A DWT Based Approach for Image Steganography,” no. 4, pp. 275-290, 2006.

[49] D. Wu and W. Tsai, “A steganographic method for images by pixel-value differencing,” vol. 24, pp. 1613-1626, 2003.

[50] C. López, "Watermarking of digital geospatial datasets: a review of technical, legal and copyright issues," Int. J. Geogr. Inf. Sci., vol. 16, no. 6, pp. 589607,2002

[51] R. Mukherjee and N. Ghoshal, "Steganography Based Visual Cryptography (SBVC)," pp. 559-566, 2013.

[52] A. Kumar and R. Sharma, "International Journal of Advanced Research in A Secure Image Steganography Based on RSA Algorithm and Hash-LSB Technique,” Int. J. Adv. Res. Comput. Sci. Softw. Eng., vol. 3, no. 7, pp. 363-372, 2013. 